\title{
Numerical Simulation of Time Fractional BBM-Burger Equation Using Cubic B-Spline Functions
}

\author{
Mohsin Kamran, ${ }^{1}$ Muhammad Abbas $\mathbb{D}^{2},{ }^{2}$ Abdul Majeed $\left(\mathbb{D},{ }^{1}\right.$ Homan Emadifar $(\mathbb{D})^{3}$ \\ and Tahir Nazir ${ }^{2}$ \\ ${ }^{1}$ Department of Mathematics, Division of Science and Technology, University of Education, Lahore, Pakistan \\ ${ }^{2}$ Department of Mathematics, University of Sargodha, 40100 Sargodha, Pakistan \\ ${ }^{3}$ Department of Mathematics, Islamic Azad University, Hamedan Branch, Hamedan, Iran
}

Correspondence should be addressed to Homan Emadifar; homan_emadi@yahoo.com

Received 18 July 2021; Accepted 20 December 2021; Published 18 January 2022

Academic Editor: Alexander Meskhi

Copyright (c) 2022 Mohsin Kamran et al. This is an open access article distributed under the Creative Commons Attribution License, which permits unrestricted use, distribution, and reproduction in any medium, provided the original work is properly cited.

\begin{abstract}
The unidirectional propagation of long waves in certain nonlinear dispersive system is explained by the Benjamin-Bona-MahonyBurger (BBM-Burger) equation. The purpose of this study is to investigate the BBM-Burger equation numerically using Caputo derivative and B-spline basis functions. The fractional derivative is considered in Caputo form, and $L 1$ formula is used for discretization of temporal derivative. The interpolation of space derivative is done with the help of B-spline functions. The effect of $\alpha$ and time on solution profile of travelling wave for different domain of $x$ is discussed in this paper. The numerical results have been presented to show that the cubic B-spline method is effective and efficient in solving the time fractional Benjamin-Bona-Mahony-Burger (BBM-Burger) equation. Moreover, the convergence and stability of the proposed scheme are analyzed. The error norms are also calculated to check the accuracy of the proposed scheme. The numerical results reflect that the proposed scheme can be used for linear and highly nonlinear models.
\end{abstract}

\section{Introduction}

The fractional calculus has a long history, beginning on 30 September, 1695 [1]. Fractional derivatives are an excellent method for explaining the memory and genetic characteristics of various materials and processes. Today, fractional differential equation (FDE) plays an important role in number of fields such as mathematics and dynamic systems [2]. Noninteger-order derivatives and integrals have proved to be more useful in formulating certain electrochemical problems than conventional models [3]. Fractional partial differential equations (FPDEs) provide a powerful tool for modeling a variety of potentially emerging phenomena with a wide range of applications across applied sciences, engineering, and physics. It is the classical generalization of the calculation that involves operators of integration of noninteger orders and differentiation. They are considered to be valuable and efficient tools in both theoretical and techno- logical fields to tackle the complexity and nonlinearity of specific problems, including chemical science, fluid flow, fiber optics, signaling system, polymers, device recognition, and elastic materials $[4,5]$. A great deal of attention has been paid to managing fractional differential equations (FDEs). Specifically, fractional-order partial differential equations (PDEs) are increasingly described in viscoelasticity, mathematical biology, finance [6-8], airplane designing, traffic, population, and particle chemistry [9-11]. Elasticity, plasma, fluid mechanics, optical fiber, and other disciplines of mathematics and physics involve wave phenomena. The Benjamin-Bona-Mahony-Burger (BBM-Burger) equation, which addresses dissipation as well as nonlinearity and dispersion in the simulation of unidirectional plane waves, is defined as follows [12]:

$$
\phi_{t}-\phi_{x x t}-\alpha \phi_{x x}+\phi \phi_{x}+\beta \phi_{x}=0,(x, t) \varepsilon \Omega,
$$


subject to IC: $\phi(x, 0)=f(x), x \varepsilon[a, b]$ and BCs as follows: $\phi$ $(a, t)=g_{1}(t), \phi(b, t)=g_{2}(t)$, where $\alpha$ and $\beta$ are positive constants and domain of $\Omega=(a, b) \times(0, T]$.

Nonlinearity, dispersive, and dissipative effects are all included in Equation (1). The terms $\phi_{x x}$ and $\phi_{x x t}$ indicate the effects of dissipation and dispersion, respectively. Cracked rock, thermodynamics, acoustic waves in aharmonic crystals [13], acoustic-gravity waves in fluids, and other applications of the Benjamin-Bona-Mahony-Burger (BBM-Burger) equation and their special cases can be found in engineering and science. Benjamin-Bona-Mahony-Burger (BBM-Burger) equation is used in the analysis of the surface waves of long wavelength in liquids, hydromagnetic waves in cold plasma, and acoustic-gravity waves in compressible fluids.

The water wave model is notoriously difficult to solve analytically. So, several investigations have been done in recent years to determine the numerical solution of water wave model (see, for example, $[14,15]$ and the references therein). Researchers introduced many different methods to develop an approximate analytical solution for the fractional differential equation and systems, such as the cubic B-spline method (CBS) by $[16,17]$ and homotopy analysis method (HAM) by [18], unified method (UM) [19], RungeKutta method [20], Hermite wavelet technique together with Newton Raphson iteration method [21], and Lie symmetry method [22]. The time fractional Benjamin-Bona-MahonyBurger (BBM-Burger) equation was proposed to discuss the dynamic physical system. The time fractional operator form of BBM-Burger equation used in [23] is

$$
D_{t}^{\alpha} \phi-\phi_{x x t}+\phi_{x}+\left(\frac{\phi^{2}}{2}\right)_{x}=0, t>0, x \varepsilon I \subseteq R, \alpha \varepsilon(0,1],
$$

where $\alpha$ is the fractional order of the equation. The initial and boundary conditions are

$$
\begin{gathered}
\phi(x, 0)=\sec h^{2}\left(\frac{x}{4}\right), \\
g_{1}=\phi(a, t)=\sec h^{2}\left(\frac{a}{4}-\frac{t}{4}\right), g_{2}=\phi(b, t)=\sec h^{2}\left(\frac{b}{4}-\frac{t}{4}\right), a, b \varepsilon Z,
\end{gathered}
$$

respectively. The exact solution of Equation (2) for $\alpha=1$ is

$$
\phi(x, t)=\sec h^{2}\left(\frac{x}{4}-\frac{t}{4}\right) .
$$

Many scholars contributed by developing new analytical and numerical schemes to solve fractional model of BBMBurger. Zhang et al. [23] implemented modified residual power series method (RPSM) for the simulations of BBMBurger equation. $G^{\prime} / G$ expansion method has been implemented to investigate the exact solution of time fractional model of BBM-Burger equation in [24]. The authors in [25] utilized Lie symmetry approach to develop solution of fractional BBM equation. New soliton solutions for $(3+1)$ -dimensional Extended Date-Jimbo-Kashiwara-Miwa Equation has been established by means of Hirota method together with quadratic test functions in [26]. In [27], the authors studied numerical solution of $(3+1)$-dimensional shallow water wave equation and explicit traveling wave solutions for Zoomeron evolution equation using Lie vector technique. Ali et al. $[28,29]$ developed new method based on hybrid orthonormal Bernstein and block-pulse function wavelet method (HOBW) for the solution of fractional voltera-Fredholm differential equation and for the solution of 2D Bratu problem. Runge-Kutta (RK4) method and Keller-Box scheme have been implemented for the analysis of the melting heat transport of blood with time-dependent cross nanofluid model by [30]. The authors in [31] developed the Gudermannian neural networks to solve the nonlinear fifth-order induction motor model (FO-IMM). Ali [32, 33] analyzed heat transfer of Ostwald-de Waele fluid using method of lines and penalty-artificial compressibility technique (PACT) for studying free convection of magnetohydrodynamic unsteady incompressible flow in a square cavity, respectively. New homotopy analysis transform method has been established in [34] to constitute analytical solution of BBM-Burger equation. The exact solution of BBM-Burger equation of fractional order has been developed by HAM in [12]. The author in [35] investigated the solution of Benjamin-Bona-Mahony equation using linearized difference scheme. The linear difference scheme with CrankNicolson has been implemented in [36] for the treatment of Benjamin-Bona-Mahony equation with a time fractional nonlocal viscous term. Majeed et al. [37] employed cubic $\mathrm{B}$-spline scheme for the approximate solution of time fractional nonhomogeneous BBM-Burger equation. [38] used Pohozhaev nonlinear capacity method to investigate the blowing-up solutions of different nonlinear fractional partial differential equations like $\mathrm{KdV}$, time fractional modified KdV-Burger, BBM-Burger, Rosenau, Ostrovsky, and Degasperis-Procesi. [39] has investigated the analytical solution of time fractional Benjamin-Bona-Mahony equation using method based on Laplace Transformation and Adomian decomposition method. Two problems have been solved with different initial conditions. In [40], the authors have derived the Benjamin-Bona-Mahony and KdV equations from water wave model. Further travelling wave equations of these models have been derived by two different methods. Space-time fractional BBM-Burger models have been solved analytically using Adomian decomposition method by [41]. The energy method is used by [42] to prove the uniqueness and existence of BBM-Burger model. The authors also used the Fourier Spectral method to get numerical solution of this model. In [43], the authors used EMAEM method to get different solutions of 3D fractional WBBM models. The sine-cosine function method is used in [44] to investigate the analytical solution of Benjamin-BonaMahony and MRLW models. The modified simple method and $G / G$ expansion method have been used in [45] to find the exact solution of TFBBM equations.

In this study, cubic B-spline method along with Caputo derivative will be used to solve time fractional BBM-Burger equation numerically. $L 1$ formula will be used to tackle the noninteger-order derivative, while B-spline basis functions will be used to discretize the space derivative. The 
advantages of B-spline collocation method over the existing schemes are that it efficiently delivers a piecewise-continuous, closed form solution and it is simpler and can be used to a wide range of problems involving partial and fractional partial differential equations. The advantage of this method over others is that once the solution has been computed, the information needed for interpolation at different locations in the interval is available. The key benefit of this method is that it can approximate the analytical curve to a given degree of smoothness. As a result, the spline approach, when compared to previous methods, has the flexibility to obtain an estimate at any point in the domain with more accurate results. The behavior of solution profile for different domains of $x$ will be studied, and the effect of parameter $\alpha$ will be discussed for various values. The objective of this study is to solve nonlinear fractional-order partial differential equation with mixed partial derivative numerically using scheme based on cubic B-spline bases. We proved that the proposed scheme is unconditionally stable.

The organization of the paper is as follows: Section 2 gives brief introduction and preliminaries. Section 3 presents the algorithm and implementation of the suggested scheme to solve the fractional model of BBM-Burger. The stability and convergence of the proposed scheme are analyzed in Sections 4 and 5, respectively. Discussion on results is presented in Section 6, and Section 7 reviews the concluding remarks.

\section{Basic Definitions}

Definition 1. The $\alpha^{\text {th }}$-order Caputo derivative [3] is defined as

$$
\frac{\partial^{\alpha} \phi(x, t)}{\partial t^{\alpha}}=\left\{\begin{array}{l}
\frac{1}{\Gamma(l-a)} \int_{0}^{t} \frac{\partial^{l} \phi(x, s)}{\partial t^{l}}(t-s)^{l-\alpha-1} d s, l-1<\alpha<l, \\
\frac{\partial^{l} \phi(x, t)}{\partial t^{l}} l=\alpha .
\end{array}\right.
$$

Definition 2. The Caputo derivative is discretized by $L 1$ formula [46]

$$
\frac{\partial^{\alpha} \phi}{\partial t^{\alpha}} \mid t_{n}=\frac{1}{(\Delta t)^{\alpha} \Gamma(2-\alpha)} \sum_{\wp=0}^{n-1} \aleph_{\wp}\left[\phi\left(t_{n-\wp}\right)-\phi\left(t_{n-\wp+1}\right)\right]+O(\Delta t)
$$

Lemma 3. The coefficients $\aleph_{\wp}=(\wp+1)^{1-\alpha}-\wp^{1-\alpha}$ and $\omega=\left(t_{n+1}-s\right)$ in Equation (6) satisfy the following properties [16]:

(i) $\aleph_{\wp}>0, \wp=0,1,2, \cdots, n$

(ii) $1=\aleph_{0}>\aleph_{1}>\aleph_{2}>\cdots>\aleph_{n}, \aleph_{n} \longrightarrow 0, n \longrightarrow \infty$

(iii) $\sum_{\wp=0}^{n}\left(\aleph_{\wp}-\aleph_{\wp+1}\right)=\left(1-\aleph_{0}\right)+\sum_{\wp=1}^{n-1} \aleph_{n}=1$
Definition 4. The generalized linearization formula used in [47] is applied in this work.

$$
\left(\phi^{\rho} \phi_{x}\right)_{j}^{n+1}=\rho \phi_{j}^{n+1}\left((\phi)^{\rho-1} \phi_{x}\right)_{j}^{n}+\left(\phi_{x}\right)_{j}^{n+1}\left(\phi^{\rho}\right)_{j}^{n}-\rho\left(\phi^{\rho} \phi_{x}\right)_{j}^{n} .
$$

Here, $\rho$ is for positive integer.

\section{Proposed Scheme}

To implement the numerical schemes, let $[0, T]$ be the domain for time and is splitted up into $N$ uniformly sized subintervals $\left[t_{n}, t_{n+1}\right]: t_{n}=n \Delta t, n=0, \cdots, N$ where $\Delta t=T / N$. The time fractional derivative in Equation (5) for $l=1$ can be evaluated at $t=t_{n+1}$ by the formula [46]. Using Equation (6) together with forward difference scheme and Crank-Nicolson for time derivative, Equation (2) becomes the following:

$$
\begin{aligned}
\gamma & \sum_{\wp=0}^{n} \aleph_{\wp}\left[\phi^{n-\wp+1}-\phi^{n-\wp}\right]-\frac{\phi_{x x}^{n+1}-\phi_{x x}^{n}}{\Delta t}+\frac{\phi_{x}^{n+1}-\phi_{x}^{n}}{2} \\
& +\frac{\left(\phi \phi_{x}\right)^{n+1}+\left(\phi \phi_{x}\right)^{n}}{2}=0
\end{aligned}
$$

where $\phi^{n}=\phi(x, t)$ and $\gamma=1 /(\Delta t)^{\alpha} \Gamma(2-\alpha)$. Replacing Equation (7) to Equation (8), we get

$$
\begin{aligned}
& 2 \Delta t \gamma \phi^{n+1}-2 \phi_{x x}^{n+1}+\Delta t \phi_{x}^{n+1}+\Delta t\left[\phi^{n+1} \phi_{x}^{n}+\phi^{n} \phi_{x}^{n+1}\right] \\
& \quad=2 \Delta t \gamma \phi^{n}-2 \phi_{x x}^{n}-\Delta t \phi_{x}^{n}-\gamma \sum \aleph_{\wp}\left[\phi^{n-\wp+1}-\phi^{n-\wp}\right] .
\end{aligned}
$$

To apply the proposed scheme, the domain of spatial variable $[0, L]$ is partitioned into $N$ subdomain by $\left[x_{r+1}, x_{r}\right]$, $: x_{r}=r h$, where $h=L / N$ and $r=0, \cdots, N$. The solution of Equation (9) at $(n+1)^{\text {th }}$ time step is given by

$$
\phi_{N}(x, t)=\sum_{j=-1}^{N+1} \Psi_{j}(t) \Lambda_{j}(x)
$$

where $\Psi_{j}\left(t_{n+1}\right)^{\prime} s$ are unknowns to be determined and $\Lambda_{j}$ $(x)$ are spline function basis of third degree defined as follows [16]:

$$
\Lambda_{j}(x)=\frac{1}{6 h^{3}}\left\{\begin{array}{l}
\left(x-x_{j}\right)^{3}, \text { if } x \in\left[x_{j}, x_{j+1}\right), \\
h^{3}+3 h^{2}\left(x-x_{j+1}\right)+3 h\left(x-x_{j+1}\right)^{2}-3\left(x-x_{j+1}\right)^{3}, \text { if } x \in\left[x_{j+1}, x_{j+2}\right] \\
h^{3}+3 h^{2}\left(x_{j+3}-x\right)+3 h\left(x_{j+3}-x\right)^{2}-3\left(x_{j+3}-x\right)^{3}, \text { if } x \in\left[x_{j+2}, x_{j+3}\right], \\
\left(x_{j+4}-x\right)^{3} \text {.if } x \in\left[x_{j+3}, x_{j+4}\right) .
\end{array}\right.
$$

Different derivatives like $\phi, \phi^{\prime}$, and $\phi^{\prime \prime}$ of Equation (10) in terms of $\Psi_{j}$ at knot points can be expressed as follows: 


$$
\begin{gathered}
\phi\left(x_{j}\right)=\left[\frac{\left(\Psi_{j-1}(t)+4 \Psi_{j}(t)+\Psi_{j+1}(t)\right)}{6}\right], \\
\phi^{\prime}\left(x_{j}\right)=\left[\frac{\left(-\Psi_{j-1}(t)+\Psi_{j+1}(t)\right)}{2 h}\right], \\
\phi^{\prime \prime}\left(x_{j}\right)=\left[\frac{\left(\Psi_{j-1}(t)-2 \Psi_{j}(t)+\Psi_{j+1}(t)\right)}{h^{2}}\right] .
\end{gathered}
$$

The variation of $\phi_{N}(x, t)$ over the typical component $\left[x_{j}, x_{j+1}\right]$ is given by

$$
\phi_{N}(x, t)=\sum_{r=j-1}^{j+2} \Psi_{r}(t) \Lambda_{r}(x) .
$$

On substituting Equation (10) and its necessary derivatives of Equation (12) in Equation (9), we obtain the following set of equations:

$$
\begin{aligned}
& \Psi_{r-1}^{n+1}\left(\chi_{0}\right)+\Psi_{r}^{n+1}\left(\chi_{1}\right)+\Psi_{r+1}^{n+1}\left(\chi_{2}\right)=\Psi_{r-1}^{n}\left(G_{1}\right)+\Psi_{r}^{n}\left(G_{2}\right)+\Psi_{r+1}^{n}\left(G_{3}\right) \\
& -\gamma \sum_{\mathfrak{\wp}=1}^{n-1} \aleph_{\mathfrak{\wp}}\left[\frac{\left(\left(\Psi_{r-1}^{n-\beta+1}-\Psi_{r-1}^{n-p}\right)+4\left(\Psi_{r}^{n-\beta+1}-\Psi_{r}^{n-\mathcal{Q}}\right)+\left(\Psi_{r+1}^{n-\beta+1}-\Psi_{r+1}^{n-p}\right)\right)}{6}\right] \text {, }
\end{aligned}
$$

where

$$
\begin{gathered}
\chi_{0}=\frac{\gamma}{6}-\frac{1}{h^{2} \Delta t}+\frac{1}{24 h}\left(Z^{\star}\right)-\frac{1}{24 h}\left(Z^{\star \star}\right)-\frac{1}{4 h}, \\
\chi_{1}=\frac{4 \gamma}{6}+\frac{2}{h^{2} \delta t}+\frac{1}{6 h}\left(Z^{\star}\right), \\
\chi_{2}=\frac{\gamma}{6}-\frac{1}{h^{2} \Delta t}+\frac{1}{24 h}\left(Z^{\star}\right)+\frac{1}{24 h}\left(Z^{\star \star}\right)+\frac{1}{4 h}, \\
G_{1}=\frac{\gamma}{6}-\frac{1}{h^{2} \Delta t}+\frac{1}{4 h}, \\
G_{2}=\frac{4 \gamma}{6}+\frac{2}{h^{2} \Delta t}, \\
G_{3}=\frac{\gamma}{6}-\frac{1}{h^{2} \Delta t}-\frac{1}{4 h}, \\
Z^{\star \star}=\Psi_{r-1}^{n}+4 \Psi_{r}^{n}+\Psi_{r+1}^{n}, \\
Z^{\star}=\Psi_{r+1}^{n}-\Psi_{r-1}^{n}, \\
\gamma=\frac{(\Delta t)^{-\alpha}}{\Gamma(2-\alpha)} .
\end{gathered}
$$

Equation (14) will be solved for $\wp=0$ first, and then, loop will be executed for $\wp \geq 1$, which gives $N+1$ system of linear equations with $N+3$ unknowns $\left(\Psi_{1}, \cdots, \Psi_{N+1}\right)^{T}$. To get consistent solution of this system, we need two more equations, for this derivative equations will be used.

$$
\begin{gathered}
{\left[\frac{\left(\Psi_{-1}^{n+1}+4 \Psi_{0}^{n+1}+\Psi_{1}^{n+1}\right)}{6}\right]=g_{1}^{n+1},} \\
{\left[\frac{\left(\Psi_{N-1}^{n+1}+4 \Psi_{N}^{n+1}+\Psi_{N+1}^{n+1}\right)}{6}\right]=g_{2}^{n+1} .}
\end{gathered}
$$

Equations (14) and (16) have the matrix form as follows:

$$
\begin{gathered}
M \lambda^{n+1}=E \lambda^{n}, \\
\lambda=\left(\Psi_{-1}^{n+1}, \Psi_{0}^{n+1}, \Psi_{1}^{n+1}, \cdots, \Psi_{N}^{n+1}, \Psi_{N+1}^{n+1}\right)^{T},
\end{gathered}
$$

where

$$
M=\left[\begin{array}{cccccccc}
\frac{1}{6} & 4 & 1 & 0 & \cdots & 0 & 0 & 0 \\
\chi_{0}^{n} & \chi_{1}^{n} & \chi_{2}^{n} & 0 & \cdots & 0 & 0 & 0 \\
\vdots & \vdots & \vdots & \vdots & \ddots & \vdots & \vdots & \vdots \\
0 & 0 & 0 & 0 & \cdots & \chi_{0}^{n} & \chi_{1}^{n} & \chi_{2}^{n} \\
0 & 0 & 0 & 0 & \cdots & \frac{1}{6} & \frac{4}{6} & \frac{1}{6}
\end{array}\right] .
$$

The approximate solution for $(n+1)^{\text {th }}$ time level can be achieved for $n=0, \cdots, N$ by using vector $\Psi$ in Equation (10).

3.1. Initial Value. To compute the initial value $\lambda=$ $\left(\Psi_{-1}^{0}, \Psi_{0}^{0}, \Psi_{1}^{0}, \cdots, \Psi_{N^{0}}, \Psi_{N+1}^{0}\right)^{T}$, the enforced conditions of the given problem will help. So, Equation (10) becomes

$$
\phi_{N}(x, 0)=\sum_{j=-1}^{N+1} \Psi_{j}(0) \Lambda_{j}(x) .
$$

To determine $\Psi^{0}$, the derivatives of the given auxiliary conditions are approximated as follows [48].

(i) $\left(\phi_{x}\right)_{j}^{k}=f^{\prime}\left(x_{j}\right)$ for $j=0$

(ii) $(\phi)_{j}^{0}=f\left(x_{j}\right)$ for $j=0,1,2, \cdots, N$

(iii) $\left(\phi_{x}\right)_{j}^{k}=f^{\prime}\left(x_{j}\right)$ for $j=N$

This gives $(N+3) \times(N+3)$ matrix equation.

$$
\left[\begin{array}{cccccccc}
\frac{-1}{2 h} & 0 & \frac{1}{2 h} & 0 & \cdots & 0 & 0 & 0 \\
\frac{1}{6} & \frac{4}{6} & \frac{1}{6} & 0 & \cdots & 0 & 0 & 0 \\
\vdots & \vdots & \vdots & \vdots & \ddots & \vdots & \vdots & \vdots \\
0 & 0 & 0 & 0 & \cdots & \frac{1}{6} & \frac{4}{6} & \frac{1}{6} \\
0 & 0 & 0 & 0 & \cdots & \frac{-1}{2 h} & 0 & \frac{1}{2 h}
\end{array}\right]\left[\begin{array}{c}
\Psi_{-1}^{0} \\
\Psi_{0}^{0} \\
\vdots \\
\Psi_{N+1}^{0}
\end{array}\right]=\left[\begin{array}{c}
f^{\prime}\left(x_{0}\right) \\
f\left(x_{0}\right) \\
\vdots \\
f\left(x_{N}\right) \\
f^{\prime}\left(x_{N}\right)
\end{array}\right],
$$


The numerical simulations have been done with the help of the MATLAB 2015b software.

\section{Stability Analysis}

The most frequently used formula to determine the stability is von Neumann Fourier formula [47]. Solution will be con- sidered in single Fourier mode.

$$
\Psi_{j}^{n}=\Psi^{k} e^{\iota \zeta p h}, \text { where } \iota=\sqrt{-1}
$$

Using Equation (21) in Equation (14), we have

$$
\begin{aligned}
& \Psi^{\wp+1} e^{l \zeta(p-1) h}\left(\chi_{0}\right)+\Psi^{\wp+1} e^{\zeta \zeta(p) h}\left(\chi_{1}\right)+\Psi^{\wp+1} e^{\zeta \zeta(p+1) h}\left(\chi_{2}\right)=\Psi^{\wp} e^{\zeta \zeta(p-1) h}\left(G_{1}\right)+\Psi^{\wp} e^{\zeta \zeta(p) h}\left(G_{2}\right)+\Psi^{\wp} e^{l \zeta(p+1) h}\left(G_{3}\right) \\
& -\gamma \sum_{\wp=1}^{n} \aleph_{\wp}\left[\frac{\left(\left(\Psi^{n-\wp+1} e^{\zeta \zeta(p-1) h}-\Psi^{n-\wp} e^{\zeta \zeta(p-1) h}\right)+4\left(\Psi^{n-\wp+1} e^{\zeta \zeta(p) h}-\Psi^{n-\wp} e^{l \zeta(p) h}\right)+\left(\Psi^{n-\wp+1} e^{l \zeta(p+1) h}-\Psi^{n-\wp} e^{\zeta \zeta(p+1) h}\right)\right)}{6}\right], \\
& \Psi^{\wp+1} e^{-l \zeta p h}\left[e^{-l \zeta h}\left(\chi_{0}\right)+\left(\chi_{1}\right)+e^{\iota \zeta h}\left(\chi_{2}\right)\right]=\Psi^{\wp} e^{l \zeta p h}\left[e^{-\iota \zeta h}\left(G_{1}\right)+\left(G_{2}\right)+e^{l \zeta h}\left(G_{3}\right)\right] \\
& -\gamma \sum_{\wp=1}^{n} \aleph_{\wp}\left[\frac{\left(\Psi^{n-\wp+1} e^{\iota \zeta(p) h}\left(e^{-} \iota \zeta h+4+e^{\iota \zeta h}\right)-\Psi^{n-\wp} e^{\iota \zeta(p) h}\left(e^{-l \zeta h}+4+e^{\iota \zeta h}\right)\right.}{6}\right], \\
& \Psi^{\wp+1}=\frac{\Psi^{\wp}\left[e^{-\iota \zeta h}\left(G_{1}\right)+G_{2}+e^{\iota \zeta h}\left(G_{3}\right)\right]}{\left[e^{-\iota \zeta h}\left(\chi_{0}\right)+\chi_{1}+e^{\iota \zeta h}\left(\chi_{2}\right)\right]} \\
& -\frac{\gamma \sum_{\wp=1}^{n} \aleph_{\wp}\left[\left(\Psi^{n-\wp+1} e^{\zeta \zeta(p) h}\left(e^{-\iota \zeta h}+4+e^{\iota \zeta h}\right)-\Psi^{n-\wp} e^{\iota \zeta(p) h}\left(e^{-\iota \zeta h}+4+e^{\iota \zeta h}\right) / 6\right]\right.}{\left[e^{-\iota \zeta h}\left(\chi_{0}\right)+\chi_{1}+e^{\iota \zeta h}\left(\chi_{2}\right)\right]} .
\end{aligned}
$$

By putting values of $\chi_{0}, \chi_{1}, \chi_{2}$ and $G_{1}, G_{2}, G_{3}$ and after simplification, we have

$$
\Psi^{\wp+1}=\frac{\Psi^{\wp}\left[\gamma / 3(\cos \zeta h)-2 / h^{2} \Delta t(\cos \zeta h)+\left(4 \gamma / 6+2 / h^{2} \Delta t\right)\right]-\gamma \sum_{\wp=1}^{n} \aleph_{\wp} 1 / 6\left[\left(e^{-l \zeta h}+4+e^{i \zeta h}\right)\left(\Psi^{n-\wp+1}-\Psi^{n-\wp}\right)\right]}{\gamma / 3(\cos \zeta h)-2 / h^{2} \Delta t(\cos \zeta h)+Z^{\star} / 12 h(\cos \zeta h)-Z^{\star \star} / 12 h(\sin \zeta h)-1 / 2 h(\sin \zeta h)+\left(4 \gamma / 6+2 / h^{2} \Delta t+1 / 6 h\left(Z^{\star}\right)\right)},
$$

where $Z^{\star}, Z^{\star \star}$, and $\gamma$ were defined above. If augment factor $\left|\Psi^{\wp+1}\right| \leq 1$, then the suggested scheme will be stable. Otherwise, it will be unstable.

$$
\begin{gathered}
\Psi^{\wp+1} \leq \Psi^{\wp}, \\
\Psi^{\wp+1} \leq 1 .
\end{gathered}
$$

The above result indicates that the proposed scheme is stable unconditionally.

\section{Convergence Analysis}

Let $\phi(x, t)$ be the exact solution of Equation (2). Assume that $G>0$ which takes different values at various locations as in [49] and is independent of $j, n, h$, and $\chi$ with the smoothness conditions that

$$
\left|\phi_{t t}\right| \leq G,\left|\phi_{x x x x}\right| \leq G \text {, for all }(x, t) \in \Omega_{h} * \Omega_{\chi} .
$$

We first linearize the term $\phi_{x}$ by taking $\phi_{x}$ as a constant " $b$." The linearized form of the proposed scheme is then

$$
\begin{aligned}
& \frac{1}{\lambda_{0}} \sum\left[\left((l+1)^{1-\alpha}-l^{1-\alpha}\right)\left(\phi^{n-l+1}-\phi^{n-l}\right)\right] \\
& -\left(\phi_{j}^{n+1}\right)_{x x t}+b=-(\phi)_{j}^{n+1}(b),
\end{aligned}
$$

where $\lambda_{0}=(\Delta t)^{\alpha} \Gamma(2-\alpha)$.

After simplifying Equation (26), we get

$$
\phi_{j}^{n+1}=\kappa\left[\sum w_{l} \phi_{j}^{n-l}-\alpha_{0}\left(\phi_{j}^{n+1}\right)_{x x t}+b\right]
$$

where $\kappa=1 /-d, \quad w_{l}=(l+1)^{1-\alpha}-l^{1-\alpha}$, and $\phi_{j}^{n-1}=\phi^{n-l+1}-$ $\phi^{n-l}$. Using Equation (25), for the scheme Equation (27), we have 


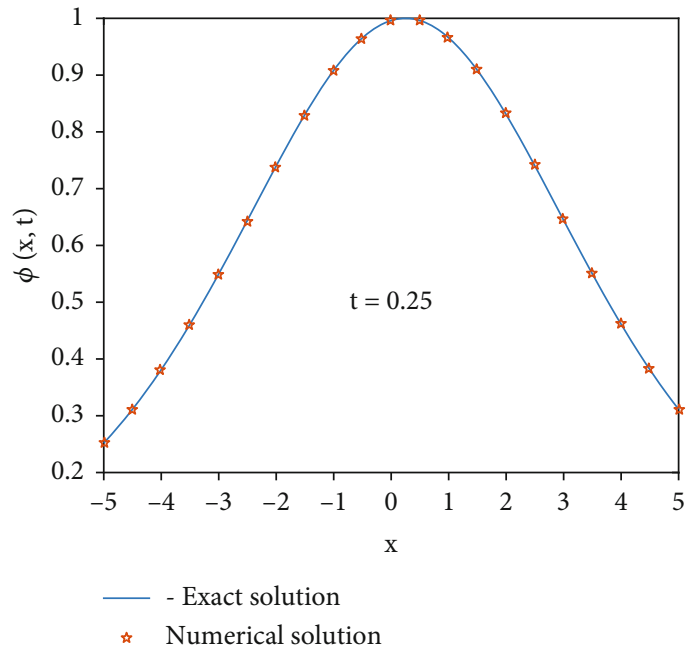

(a) For $t=0.25$

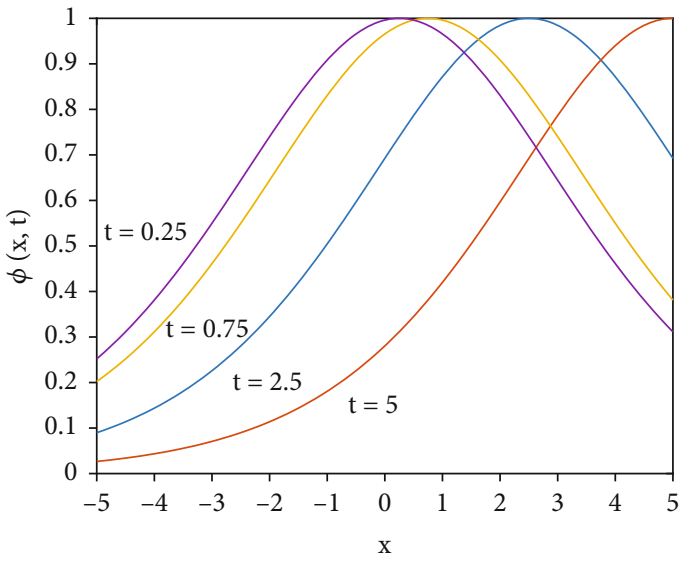

(b) For $t=0.25,0.75,2.5$, and 5

FIgURE 1: Exact and numerical solution of time fractional BBMB-equation for $\alpha=0.95, \Delta t=0.0 .00943, \beta=1$, and $h=0.01$.

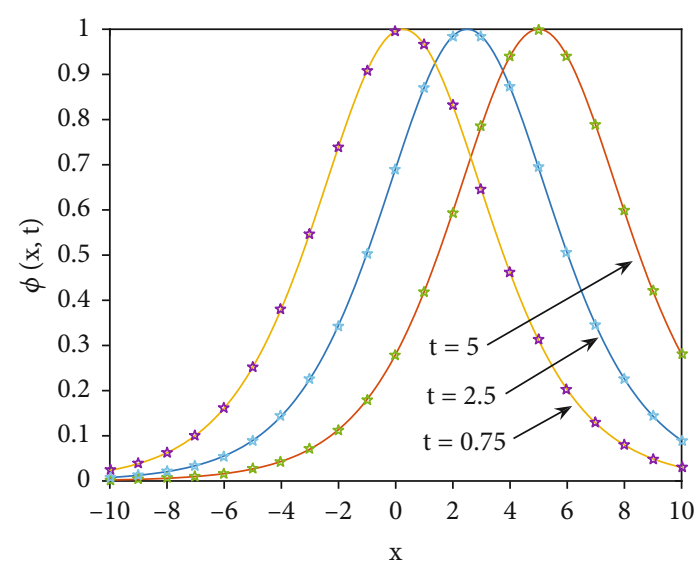

FIgURE 2: Exact and numerical results of time fractional BBMBequation for $t=0.75,2.5$, and $5, \alpha=0.5, \Delta t=0.011, \beta=1$, and $h=0.01$.

$\phi\left(x_{j}, t^{n+1}\right)=\kappa\left[\sum w_{l} \phi\left(x_{j}, t^{n-l}\right)-\alpha_{0} \phi\left(x_{j}, t^{n+1}\right)_{x x t}+b\right]+O\left(\chi^{2}+\chi h^{2}\right)$

where $\phi\left(x_{j}, t^{n}\right)$ is the exact solution at point $\left(x_{j}, t^{n}\right)$.

$$
\left\|g^{n+1}\right\| \leq G \kappa+O\left(\chi^{2}+\chi h^{2}\right)
$$

where $g_{j}^{n+1}=\phi\left(x_{j}, t^{n+1}\right)-\left(\phi_{j}^{n+1}\right.$ and $G$ is a constant.

Theorem 5. If $\phi(x, t)$ and $\phi_{j}^{n}$ are the solutions of given equation and Equation (27), respectively, and if $\phi(x, t)$ satisfies smoothness condition Equation (25), then for sufficiently small $h$ and $\chi$,

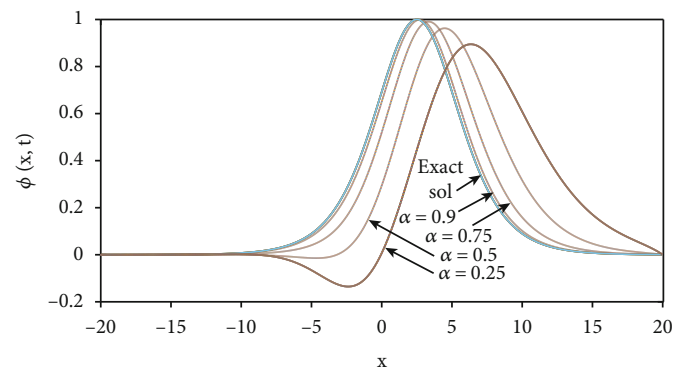

FIgURE 3: Effect of $\alpha \varepsilon(0,1)$ of time fractional BBMB-equation for $x \varepsilon[-10,10], \Delta t=0.011, \beta=1$, and $h=0.01$

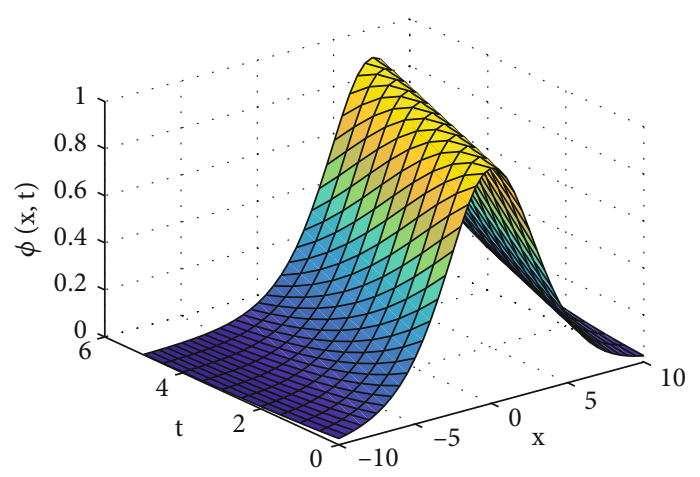

Figure 4: 3D image of numerical results of time fractional BBMBequation for $x \varepsilon[-10,10], \alpha=0.5, \Delta t=0.011, \beta=1$, and $h=0.01$

Proof. Subtracting Equation (27) from Equation (28), we obtain

$$
g_{j}^{n+1}=\kappa\left[\sum w_{l} g_{j}^{n-l}-\lambda_{0}\left(g_{j}^{n+1}\right)_{x x t}\right]+s_{j}^{n+l}
$$

where $s_{j}^{n+l}=O\left(\chi^{2}+\chi h^{2}\right)$. Multiplying the above equation by $g_{j}^{n+1}$ and summing over $j$ from 1 to $M$, we obtain 
TABLE 1: Exact and approximate results for various time levels with $\alpha=0.95$.

\begin{tabular}{lccccccc}
\hline \multirow{2}{*}{$t=0.25$} & \multicolumn{2}{c}{$t=0.75$} & \multicolumn{2}{c}{$t=2.5$} & \multicolumn{2}{c}{$t=5$} \\
Exact $^{\star}$ & Approximate & Exact $^{*}$ & Approximate & Exact $^{\star}$ & Approximate & Exact $^{*}$ Approximate $^{*}$ \\
\hline 0.00016024 & 0.000143214 & 0.00012480 & 0.00013210 & 0.00005202 & 0.00005432 & 0.00004906 & 0.00005431 \\
0.00117757 & 0.00121347 & 0.00091721 & 0.00093214 & 0.00038245 & 0.00038321 & 0.00010959 & 0.00010991 \\
0.00866854 & 0.00867653 & 0.00675756 & 0.00674301 & 0.00282253 & 0.00286532 & 0.00080948 & 0.00080321 \\
0.06231111 & 0.06232143 & 0.04886955 & 0.04883210 & 0.02066898 & 0.02066321 & 0.00596590 & 0.00596987 \\
0.37973346 & 0.37974321 & 0.31013874 & 0.31013311 & 0.14307075 & 0.14304098 & 0.04325287 & 0.04325138 \\
0.99578687 & 0.99570213 & 0.96475136 & 0.96471098 & 0.69049874 & 0.69043210 & 0.27922753 & 0.27922665 \\
0.46283332 & 0.46289872 & 0.55164888 & 0.55163211 & 0.87313830 & 0.87317653 & 0.93885890 & 0.93885321 \\
0.08005885 & 0.08001242 & 0.10161478 & 0.10163111 & 0.22690063 & 0.22694321 & 0.59848119 & 0.59848542 \\
0.01122801 & 0.01123201 & 0.01439402 & 0.01433201 & 0.03418321 & 0.03419807 & 0.11434897 & 0.11434873 \\
0.00152697 & 0.00151132 & 0.00196024 & 0.00196031 & 0.00469593 & 0.00467651 & 0.01629489 & 0.01629659 \\
0.00020575 & 0.00023214 & 0.00026419 & 0.00026435 & 0.00063364 & 0.00060098 & 0.00220989 & 0.00220098 \\
\hline
\end{tabular}

$\left\|g^{n+1}\right\|^{2}=\kappa\left[\sum w_{l}\left(g^{n-l}, g^{n+1}\right)-\lambda_{0}\left(\left(g^{n+1}\right)_{x x t}, g^{n+1}\right)\right]+\left(s^{n+l}, e^{n+1}\right)$.

Using the inequality $<r, t>\leq\|r\|\|t\|$, we obtain

$\left\|g^{n+1}\right\|^{2} \leq \kappa\left[\sum w_{l}\left\|g^{n-l}\right\|\left\|g^{n+1}\right\|\right]-\kappa \lambda_{0}\left\|\left(g^{n+1}\right)_{x}\right\|^{2}+\left\|s^{n+l}\right\|\left\|g^{n+1}\right\|$.

Since $\kappa \lambda_{0}\left\|\left(g^{n+1}\right)_{x}\right\|^{2} \geq 0$, therefore

$$
\left\|g^{n+1}\right\|^{2} \leq \kappa\left[\sum w_{l}\left\|g^{n-l}\right\|\left\|g^{n+1}\right\|\right]+\left\|s^{n+l}\right\|\left\|g^{n+1}\right\|
$$

Dividing Equation (33) throughout by $\left\|g^{n+1}\right\|$, we get

$$
\begin{aligned}
\left\|g^{n+1}\right\| & \leq \kappa\left[\sum w_{l}\left\|g^{n-l}\right\|\right]+\left\|s^{n+l}\right\|, \\
& \leq \kappa G\left[\sum w_{l}\right]+\left\|s^{n+l}\right\|, \\
& <\kappa G+O\left(\chi^{2}+\chi h^{2}\right),
\end{aligned}
$$

where $G=\max (\max )\left\|g^{n-l}\right\|$. Note that we have used $s_{j}^{n+l}=$ $O\left(\chi^{2}+\chi h^{2}\right)$. This completes the proof.

\section{Discussion}

The numerical results obtained by the suggested scheme for various parameters are reported in this part in the form of tables and figures. $L_{2}$ and $L_{\infty}$ norms are computed and defined as

$$
\begin{gathered}
L_{2}=\left\|\phi_{\text {exact }^{\star}}-\phi_{\text {approx }}\right\|_{2} \simeq \sqrt{h \sum_{p=0}^{n}\left|\left(\phi_{p}\right)_{\text {exact }^{\star}}-\left(\phi_{p}\right)_{\text {approx }}\right|^{2}}, \\
L_{\infty}=\left\|\phi_{\text {exact }^{\star}}-\phi_{\text {approx }}\right\|_{\infty} \simeq \max _{p}\left|\left(\phi_{p}\right)_{\text {exact }^{\star}}-\left(\phi_{p}\right)_{\text {approx }}\right| .
\end{gathered}
$$

TABLE 2: Error norms.

\begin{tabular}{lccc}
\hline$t$ & $L_{2}$ norm & $L_{\infty}$ norm & CPU time \\
\hline 0.25 & $1.281 \times 10^{-5}$ & $8.474 \times 10^{-5}$ & \\
0.75 & $8.913 \times 10^{-6}$ & $6.201 \times 10^{-5}$ & 1.75 \\
2.5 & $1.101 \times 10^{-5}$ & $6.664 \times 10^{-5}$ & \\
5 & $1.478 \times 10^{-6}$ & $8.91 \times 10^{-6}$ & \\
\hline
\end{tabular}

The approximate solution (10) can be written in piecewise form as follows:

$$
\begin{gathered}
\phi\left(x, t_{n}\right)=\Phi_{j-3} \Lambda_{3, j-3}(x)+\Phi_{j-2} \Lambda_{3, j-2}(x)+\Phi_{j-1} \Lambda_{3, j-1}(x)+\Phi_{j} \Lambda_{3, j}(x), x \in\left[x_{j}, x_{j+1}\right) . \\
\phi_{N}(x, 1)= \begin{cases}x^{3}-0.64 x^{2}-0.805 x+0.99997, & x \in[0,0.1), \\
0.56667 x^{3}-0.51 x^{2}-0.818 x+1.0004, & x \in[0.1,0.2), \\
16.75 x^{3}-10.22 x^{2}+1.124 x+0.87093, & x \in[0.2,0.3), \\
0.11667 x^{3}-0.195 x^{2}-0.8945 x+1.0068, & x \in[0.3,0.4), \\
-0.033333 x^{3}-0.015 x^{2}-0.9665 x+1.0165, & x \in[0.4,0.5), \\
-0.066667 x^{3}+0.035 x^{2}-0.9915 x+1.0206, & x \in[0.5,0.6), \\
-0.05 x^{3}+0.005 x^{2}-0.9735 x+1.017, & x \in[0.6,0.7), \\
-0.033333 x^{3}-0.03 x^{2}-0.949 x+1.0113, & x \in[0.7,0.8), \\
-3546.6 x^{3}+8511.7 x^{2}-6810.3 x+1816.8, & x \in[0.8,0.9), \\
10640.0 x^{3}-29793.0 x^{2}+27664.0 x-8525.4, & x \in[0.9,1) .\end{cases}
\end{gathered}
$$

The numerical solution $\phi(x, t)$ of travelling wave models is discussed graphically and numerically in our computational work, the values of the parameter $\alpha=0.95$, step length $h=0.01$, and $\Delta t=0.00943$ are chosen, and for $t=0.25$ to $t$ $=5$ computation, it was carried out over different finite space range of $x \varepsilon[-5,5], x \varepsilon[-10,10]$, and $x \varepsilon[-20,20]$. Figure 1 shows the wave evolution for different time with $\alpha$ $=0.95$. Figure $1(\mathrm{a})$ depicts the behavior of travelling wave at $t=0.25$, while Figure 1(b) demonstrates the identical patron of travelling waves for different time $t=0.25,0.75$, 2.5 , and 5. The domain of $x$ varies from -5 to 5 to observe the behavior of wave, and it is clearly shown that both 


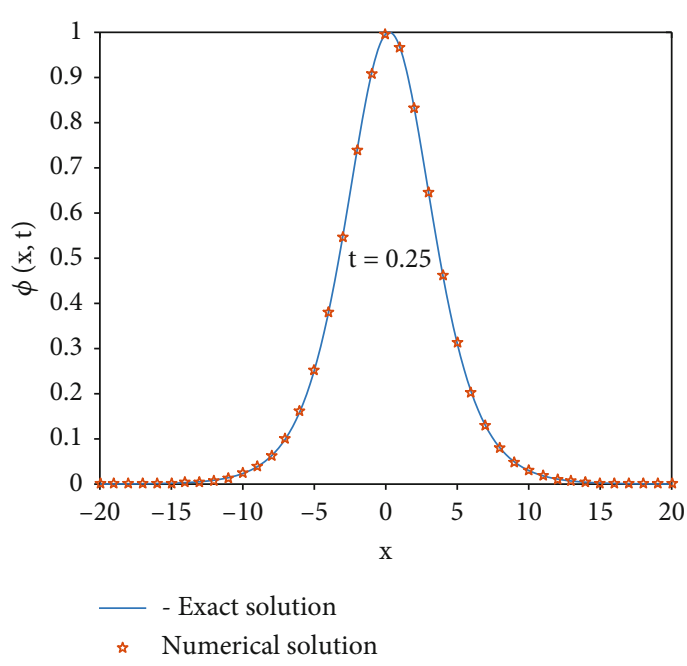

(a) For $t=0.25$

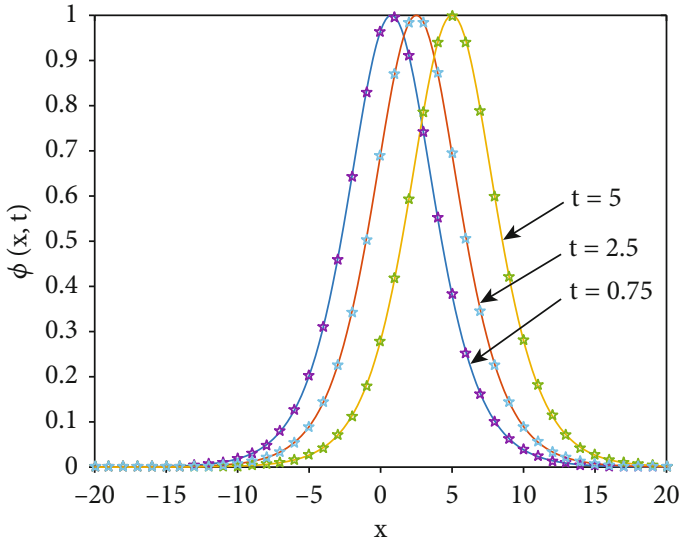

(b) For $t=0.75,2.5$, and 5

Figure 5: Approximate and classical results of time fractional BBMB-equation for $\alpha=0.5, \Delta t=0.01, \beta=1$, and $h=0.01$.

solutions are in good consensus with each other with identical patron of waves.

The graphical illustration displayed in Figure 2 expresses the behavior of solution profile for different time steps $t=$ $0.75,2.5$, and 5 in broader domain of $x \varepsilon[-10,10]$ and keeping $\alpha, h$, and $\Delta t=0.011$ fixed. Figure 2 express the behavior of wave evolution at $t=0.75,2.5$, and 5 in wide domain of $x \varepsilon[-10,10]$, while keeping $h, \alpha$, and $\Delta t=0.011$ fixed in this case. As the number of iterations is raised, the solution profile produces better results, and the traveling wave gets smoother in a larger domain. The wave evolution is also observed at different values of $\alpha \varepsilon(0,1]$. It is observed that as the value of $\alpha$ approaches to 1 , the numerical solution tends toward the exact ${ }^{\star}$ solution, and if we use the alpha value near 0 , numerical value moves far away from theexact ${ }^{\star}$ solution as shown in Figure 3. The 3D form of numerical solution with different time level is shown in Figure 4 effect of $\alpha$ $\varepsilon(0,1]$ is observed. The efficiency of the proposed scheme in tabular form for this model can be observed by Table 1. In this table, numerical and exact ${ }^{\star}$ solutions are compared. Table 2 presents the $L_{2}$ and $L_{\infty}$ norms.

To study the effect of solution profile on a larger domain, we extended the $x$ space domain from -20 to 20 in Figure 5. The parameters for this simulation were chosen $\alpha=0.95, h$ $=0.01$, and $\Delta t=0.01$ for different times $t=0.25,0.75,2.5$, and $t=5$. Figure 4 displays the solution profile for time $t=$ 0.25 , while the effect of different time on solution profile of travelling wave is shown in Figure 4. The graphics illustrate the smoothness of the solution profile. Figure 6 shows how different fractional parameters, such as $\alpha \varepsilon(0,1)$, affect the solution profile.

The wave swings for small values of $\alpha$ in the broader domain $x \varepsilon[-20,20]$ and then smooths out and approaches the classical solution for $\alpha \longrightarrow 1$. The numerical solution of wave model in 3D form can be seen in Figure 7. Both solutions are extremely similar to each other for different parameters, as seen in the graphics. The exact ${ }^{\star}$ and approximate outcomes are compared in Table 3. Both solutions are com-

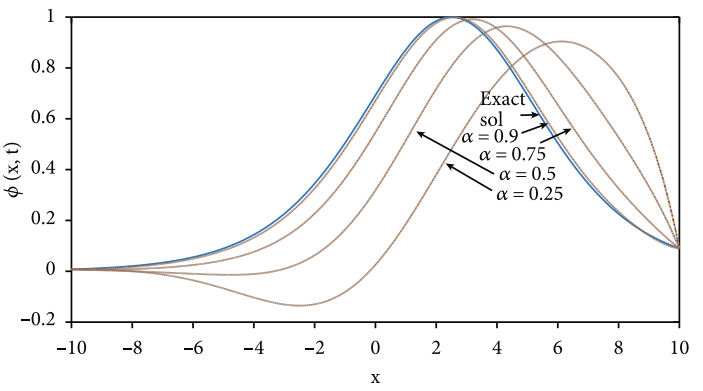

FIgURE 6: Effect of $\alpha \varepsilon(0,1)$ of time fractional BBMB-equation for $x \varepsilon[-20,20], \Delta t=0.01, \beta=1$, and $h=0.01$.

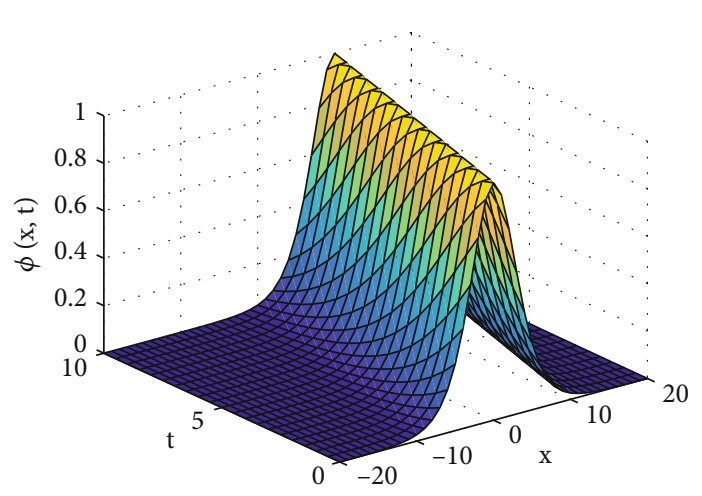

FIgURE 7: 3D image of numerical results of time fractional BBMBequation for $x \varepsilon[-20,20], \alpha=0.5, \Delta t=0.001, \beta=1$, and $h=0.01$

parable up to four decimal places, as may be shown from tabular data. The error norms obtained at $x \varepsilon[-20,20]$ are shown in Table 4. The behavior of solution profile for broader domain has been studied and illustrated in the figures. These graphics demonstrate that solution curves become smooth and swing as we extend the domain, and both results express the identical behavior. So, it is observed 
TABle 3: Comparison of outcomes at $\alpha=0.95$ for different time levels.

\begin{tabular}{|c|c|c|c|c|c|}
\hline \multicolumn{2}{|c|}{$t=0.25$} & \multicolumn{2}{|c|}{$t=2.5$} & \multicolumn{2}{|c|}{$t=5$} \\
\hline Exact $^{\star}$ & Approximate & Exact $^{\star}$ & Approximate & Exact $^{\star}$ & Approximate \\
\hline 0.02350451 & 0.02358765 & 0.00769208 & 0.00766501 & 0.00220989 & 0.00224321 \\
\hline 0.06231111 & 0.06233209 & 0.02066898 & 0.02067632 & 0.00596590 & 0.00597632 \\
\hline 0.16052847 & 0.16059852 & 0.05519448 & 0.05511096 & 0.01613407 & 0.01610987 \\
\hline 0.37973346 & 0.37972211 & 0.14307075 & 0.14304312 & 0.04325287 & 0.04325210 \\
\hline 0.73818583 & 0.73814309 & 0.34363768 & 0.34360985 & 0.11327757 & 0.11328732 \\
\hline 0.99578687 & 0.99577763 & 0.69049874 & 0.69048301 & 0.27922753 & 0.27921094 \\
\hline 0.83231679 & 0.83234312 & 0.98391831 & 0.98399901 & 0.59469199 & 0.59460073 \\
\hline 0.46283332 & 0.46284401 & 0.87313830 & 0.87316521 & 0.93885890 & 0.93881325 \\
\hline 0.20311046 & 0.20314387 & 0.50629409 & 0.50629831 & 0.94116115 & 0.94117643 \\
\hline 0.08005885 & 0.08004309 & 0.22690063 & 0.22690743 & 0.59848119 & 0.59844312 \\
\hline 0.03007930 & 0.03007915 & 0.08979764 & 0.08971487 & 0.28041486 & 0.28049875 \\
\hline
\end{tabular}

TABLE $4: L_{2}$ and $L_{\infty}$ norms.

\begin{tabular}{lccc}
\hline$t$ & $L_{2}$ norm & $L_{\infty}$ norm & CPU time \\
\hline 0.25 & $1.283 \times 10^{-5}$ & $8.314 \times 10^{-5}$ & \\
2.5 & $1.539 \times 10^{-5}$ & $8.352 \times 10^{-5}$ & 2.25 \\
5 & $1.460 \times 10^{-5}$ & $9.126 \times 10^{-5}$ & \\
\hline
\end{tabular}

that the proposed scheme gives better results for smaller and larger domains but if the spatial derivative in the model is of third or fourth order, then the proposed scheme has to be modified.

\section{Concluding Remarks}

In this work, an algorithm based on B-spline functions and Caputo derivative has been successfully implemented on fractional BBM-Burger equation. The temporal derivative is considered in Caputo's form, and discretization has been done by $L 1$ formula. The space derivative has been interpolated by means of Crank-Nicolson and B-spline function. Applicability of the scheme is examined on test problem, and the following points are extracted.

(i) The behavior of travelling wave is studied for different domains of $x$, and it is observed that as we extend the domain of $x$, the solution profile improves and wave becomes more smooth

(ii) The effect of the fractional parameter $\alpha$ on various spatial ranges has been studied, and it has been discovered that the wave varies for smaller values of $\alpha$ before becoming smooth for larger values of $\alpha$

(iii) The approximation solution tends to overlap the classical solution, as $\alpha \longrightarrow 1$ may be seen in visuals and tabular data

(iv) The study's findings on the stability of the suggested scheme reveal that it is unconditionally stable (v) The error norms for different domains of $x$ are presented in the form of table which reflects that the proposed scheme is suitable for any domain of $x$

In general, cubic B-spline scheme is an effective and convenient method in finding approximate solution for the time fractional BBM-Burger equation and other long waves in certain nonlinear dispersive systems.

\section{Data Availability}

The experimental data used to support the findings of this study are available within this paper.

\section{Conflicts of Interest}

The authors declare that they have no conflicts of interest to report regarding the present study.

\section{Authors' Contributions}

All authors equally contributed to this work. All authors read and approved the final manuscript.

\section{Acknowledgments}

We thank Dr. Muhammad Kashif Iqbal for his assistance in proofreading of the manuscript.

\section{References}

[1] K. Oldham and J. Spanier, The Fractional Calculus Theory and Applications of Differentiation and Integration to Arbitrary Order, Elsevier, 1974.

[2] A. Arikoglu and I. Ozkol, "Solution of fractional differential equations by using differential transform method," Chaos, Solitons \& Fractals, vol. 34, no. 5, pp. 1473-1481, 2007.

[3] I. Podlubny, Fractional Differential Equations, Academic Press, San Diego, 1999.

[4] S. S. Ray, "New exact solutions of nonlinear fractional acoustic wave equations in ultrasound," Computers \& Mathematics with Applications, vol. 71, no. 3, pp. 859-868, 2016. 
[5] A. Atangana and D. Baleanu, "New fractional derivatives with nonlocal and non-singular kernel: theory and application to heat transfer model," 2016, http://arxiv.org/abs/1602.03408.

[6] A. Majeed, M. Abbas, K. T. Miura, M. Kamran, and T. Nazir, "Surface modeling from 2D contours with an application to craniofacial fracture construction," Mathematics, vol. 8, no. 8, p. 1246, 2020.

[7] A. Babaei, H. Jafari, and M. Ahmadi, "A fractional order HIV/ AIDS model based on the effect of screening of unaware infectives," Mathematical Methods in the Applied Sciences, vol. 42, no. 7, pp. 2334-2343, 2019.

[8] A. Babaei, M. Ahmadi, H. Jafari, and A. Liya, "A mathematical model to examine the effect of quarantine on the spread of coronavirus," Chaos, Solitons and Fractals, vol. 142, article 110418, 2021.

[9] A. Majeed, M. Abbas, A. A. Sittar, M. Y. Misro, and M. Kamran, "Airplane designing using quadratic trigonometric B-spline with shape parameters," AIMS Mathematics, vol. 6, no. 7, pp. 7669-7683, 2021.

[10] E. Atilgan, M. Senol, A. Kurt, and O. Tasbozan, "New wave solutions of time-fractional coupled Boussinesq-WhithamBroer-Kaup equation as a model of water waves," China Ocean Engineering, vol. 33, no. 4, pp. 477-483, 2019.

[11] O. Abu Arqub, Z. Odibat, and M. al-Smadi, "Numerical solutions of time-fractional partial integrodifferential equations of Robin functions types in Hilbert space with error bounds and error estimates," Nonlinear Dynamics, vol. 94, no. 3, pp. 18191834, 2018.

[12] A. Fakhari, G. Domairry, and Ebrahimpour, "Approximate explicit solutions of nonlinear BBMB equations by homotopy analysis method and comparison with the exact solution," Physics Letters A, vol. 368, no. 1-2, pp. 64-68, 2007.

[13] S. Abbasbandy and A. Shirzadi, "The first integral method for modified Benjamin-Bona-Mahony equation," Communications in Nonlinear Science and Numerical Simulation, vol. 15, no. 7, pp. 1759-1764, 2010.

[14] B. Hu, Y. Xu, and J. Hu, "Crank-Nicolson finite difference scheme for the Rosenau-Burgers equation," Applied Mathematics and Computation, vol. 204, no. 1, pp. 311-316, 2008.

[15] J. Janwised, B. Wongsaijai, T. Mouktonglang, and K. Poochinapan, "A modified three-level average linearimplicit finite difference method for the Rosenau-Burgers equation," Adv. Math. Phys., vol. 2014, pp. 1-11, 2014.

[16] A. Majeed, M. Kamran, M. K. Iqbal, and D. Baleanu, "Solving time fractional Burgers' and Fisher's equations using cubic Bspline approximation method," Adv. Difference Equ., vol. 2020, no. 1, pp. 1-15, 2020.

[17] A. Majeed, M. Kamran, M. Abbas, and J. Singh, “An efficient numerical technique for solving time-fractional generalized Fisher's equation," Frontiers in Physics, vol. 8, p. 293, 2020.

[18] R. K. Pandey, O. P. Singh, and V. K. Baranwal, "An analytic algorithm for the space-time fractional advection-dispersion equation," Computer Physics Communications, vol. 182, no. 5, pp. 1134-1144, 2011.

[19] M. N. Rafiq, A. Majeed, S. W. Yao, M. Kamran, M. H. Rafiq, and M. Inc, "Analytical solutions of nonlinear time fractional evaluation equations via unified method with different derivatives and their comparison," Results in Physics, vol. 26, p. 104357, 2021.

[20] D. Baleanu, R. Sadat, and M. R. Ali, "The method of lines for solution of the carbon nanotubes engine oil nanofluid over an unsteady rotating disk," The European Physical Journal Plus, vol. 135, no. 10, pp. 1-13, 2020.

[21] M. R. Ali, A. R. Hadhoud, and W. X. Ma, "Evolutionary numerical approach for solving nonlinear singular periodic boundary value problems," Journal of Intelligent Fuzzy Systems, vol. 39, no. 5, pp. 7723-7731, 2020.

[22] M. R. Ali and R. Sadat, "Lie symmetry analysis, new group invariant for the $(3+1)$-dimensional and variable coefficients for liquids with gas bubbles models," Chinese Journal of Physics, vol. 71, pp. 539-547, 2021.

[23] J. Zhang, Z. Wei, L. Yong, and Y. Xiao, “Analytical solution for the time fractional BBM-Burger equation by using modified residual power series method," Complexity, vol. 2018, 11 pages, 2018.

[24] M. Shakeel, Q. M. Ul-Hassan, J. Ahmad, and T. Naqvi, "Exact solutions of the time fractional BBM-Burger equation by novel-expansion method," Adv. Math. Phys., vol. 2014, pp. 1-15, 2014.

[25] W. X. Ma, M. R. Ali, and R. Sadat, "Analytical solutions for nonlinear dispersive physical model," Complexity, vol. 2020, 8 pages, 2020.

[26] M. R. Ali and A. R. Hadhoud, "Hybrid Orthonormal Bernstein and Block-Pulse functions wavelet scheme for solving the $2 \mathrm{D}$ Bratu problem," Results in Physics, vol. 12, pp. 525-530, 2019.

[27] M. R. Ali and W. X. Ma, "Detection of new multi-wave solutions in an unbounded domain," Modern Physics Letters B, vol. 33, no. 34, p. 1950425, 2019.

[28] M. R. Ali, A. R. Hadhoud, and H. Srivastava, "Solution of fractional Volterra-Fredholm integro-differential equations under mixed boundary conditions by using the HOBW method," Adv. Difference Equ., vol. 2019, no. 1, pp. 1-14, 2019.

[29] R. Ali-M and R. Sadat, "Construction of Lump and optical solitons solutions for $(3+1)$ model for the propagation of nonlinear dispersive waves in inhomogeneous media," Optical and Quantum Electronics, vol. 53, no. 5, pp. 1-13, 2021.

[30] A. Ayub, Z. Sabir, G. C. Altamirano, R. Sadat, and M. R. Ali, "Characteristics of melting heat transport of blood with timedependent cross-nanofluid model using Keller-Box and BVP4C method," Engineering with Computers, pp. 1-15, 2021.

[31] Z. Sabir, M. A. Raja Zahor, D. Baleanu, R. Sadat, and M. Ali R., "Investigations of nonlinear induction motor model using the Gudermannian neural networks," Thermal Science, pp. 261261, 2021.

[32] M. R. Ali, "The method of lines analysis of heat transfer of Ostwald-de Waele fluid generated by a non-uniform rotating disk with a variable thickness," Journal of Applied and Computational Mechanics, vol. 7, no. 2, pp. 432-441, 2021.

[33] M. M. Mousa, M. R. Ali, and W. X. Ma, "A combined method for simulating MHD convection in square cavities through localized heating by method of line and penalty-artificial compressibility," Journal of Taibah University for Science, vol. 15, no. 1, pp. 208-217, 2021.

[34] S. Kumar and D. Kumar, "Fractional modelling for BBMBurger equation by using new homotopy analysis transform method," Journal of the Association of Arab Universities for Basic and Applied Sciences, vol. 16, no. 1, pp. 16-20, 2014.

[35] C. Li, "Linearized difference schemes for a BBM equation with a fractional nonlocal viscous term," Applied Mathematics and Computation, vol. 311, pp. 240-250, 2017.

[36] X. Shen and A. Zhu, "A Crank-Nicolson linear difference scheme for a BBM equation with a time fractional nonlocal 
viscous term," Adv. Difference Equ., vol. 2018, no. 1, pp. 1-12, 2018.

[37] A. Majeed, M. Kamran, M. Abbas, and M. Y. Bin Misro, “An efficient numerical scheme for the simulation of timefractional nonhomogeneous Benjamin-Bona-Mahony-Burger model," Physica Scripta, vol. 96, no. 8, article 084002, 2021.

[38] A. Alsaedi, B. Ahmad, M. Kirane, and B. T. Torebek, "Blowing-up solutions of the time-fractional dispersive equations," Adv. Nonlinear Anal., vol. 10, no. 1, pp. 952-971, 2021.

[39] M. Ikram, A. Muhammad, and A. Ur Rahmn, "Analytic solution to Benjamin-Bona-Mahony equation by using Laplace Adomian decomposition method," Matrix Science Mathematic, vol. 3, no. 1, pp. 01-04, 2019.

[40] A. R. Seadawy and A. Sayed, "Traveling wave solutions of the Benjamin-Bona-Mahony water wave equations," in Abstract and applied analysis (Vol. 2014), Hindawi, 2014.

[41] D. B. Dhaigude, G. A. Birajdar, and V. R. Nikam, "Adomain decomposition method for fractional Benjamin-Bona-Mahony-Burger's equations," International Journal of Applied Mathematics and Mechanics, vol. 8, no. 12, pp. 42-51, 2012.

[42] G. Oruc, H. Borluk, and G. M. Muslu, "The generalized fractional Benjamin-Bona-Mahony equation: analytical and numerical results," Physica D: Nonlinear Phenomena, vol. 409, article 132499, 2020.

[43] U. Akram, A. R. Seadawy, S. T. R. Rizvi, M. Younis, S. Althobaiti, and S. Sayed, "Traveling wave solutions for the fractional Wazwaz-Benjamin-Bona-Mahony model in arising shallow water waves," Results in Physics, vol. 20, article 103725, 2021.

[44] M. Ramli, D. Irsalina, I. P. Iwanisa, and V. Halfiani, "Soliton solution of Benjamin-Bona-Mahony equation and modified regularized long wave equation," AIP Conference Proceedings, vol. 1913, no. 1, article 020002, 2017.

[45] O. Kolebaje and O. Popoola, "Assessment of the exact solutions of the space and time fractional Benjamin- BonaMahony equation via the -expansion method, modified simple equation method, and Liu's theorem," ISRN Mathematical Physics, vol. 2014, 11 pages, 2014.

[46] A. Majeed, M. Kamran, and M. Rafique, "An approximation to the solution of time fractional modified Burgers' equation using extended cubic B-spline method," Computational and Applied Mathematics, vol. 39, no. 4, pp. 1-21, 2020.

[47] A. Majeed, M. Kamran, N. Asghar, and D. Baleanu, "Numerical approximation of inhomogeneous time fractional Burgers-Huxley equation with B-spline functions and Caputo derivative," Engineering with Computers, pp. 1-16, 2021.

[48] I. Dag, D. Irk, and B. Saka, "A numerical solution of the Burgers' equation using cubic B-splines," Applied Mathematics and Computation, vol. 163, no. 1, pp. 199-211, 2005.

[49] T. S. El-Danaf and A. R. Hadhoud, "Parametric spline functions for the solution of the one time fractional Burgers' equation," Applied Mathematical Modelling, vol. 36, no. 10, pp. 4557-4564, 2012. 